\title{
Real-time composition control of InAIAs grown on InP using spectroscopic ellipsometry
}

M. Beaudoin, E. Grassi, S. R. Johnson, K. Ramaswamy, K. Tsakalis, T. L. Alford, and Y.-H. Zhang

Citation: Journal of Vacuum Science \& Technology B 18, 1435 (2000); doi: 10.1116/1.591398

View online: http://dx.doi.org/10.1116/1.591398

View Table of Contents: http://scitation.aip.org/content/avs/journal/jvstb/18/3?ver=pdfcov

Published by the AVS: Science \& Technology of Materials, Interfaces, and Processing

\section{Articles you may be interested in}

Optical properties of Si-doped and Be-doped InAIAs lattice-matched to InP grown by molecular beam epitaxy J. Appl. Phys. 114, 103504 (2013); 10.1063/1.4820519

High-quality InAIAs layers grown on (411)A-oriented InP substrates by molecular beam epitaxy J. Vac. Sci. Technol. B 17, 1482 (1999); 10.1116/1.590778

Use of spectroscopic ellipsometry for feedback control during the growth of thin AlAs layers J. Vac. Sci. Technol. B 17, 1233 (1999); 10.1116/1.590728

Real time in situ composition control of InGaAs lattice matched to InP by an 88-wavelength ellipsometer J. Vac. Sci. Technol. B 16, 1484 (1998); 10.1116/1.589971

Ordered InAs quantum dots in InAIAs matrix on (001) InP substrates grown by molecular beam epitaxy Appl. Phys. Lett. 72, 2123 (1998); 10.1063/1.121296

\section{SHIMADZU Powerful, Multi-functional UV-Vis-NIR and Excellence in Science FTIR Spectrophotometers}

Providing the utmost in sensitivity, accuracy and resolution for applications in materials characterization and nano research

- Photovoltaics

- Polymers

- Thin films

- Paints

- Ceramics

- DNA film structures

- Coatings

- Packaging materials

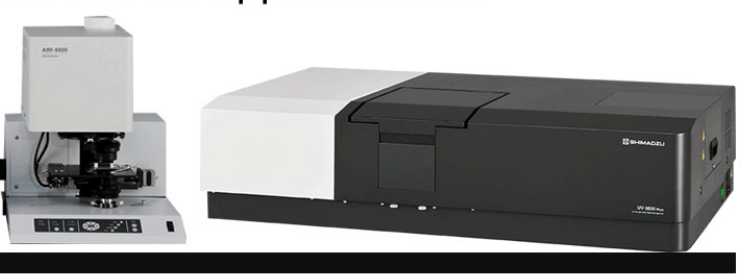




\title{
Real-time composition control of InAlAs grown on InP using spectroscopic ellipsometry
}

\author{
M. Beaudoin, ${ }^{a}$ E. Grassi, S. R. Johnson, K. Ramaswamy, K. Tsakalis, T. L. Alford, \\ and Y.-H. Zhang ${ }^{b}$ \\ Center for Solid State Electronics Research and Department of Electrical Engineering, Arizona State \\ University, Tempe, Arizona 85287-6206
}

(Received 10 October 1999; accepted 11 February 2000)

\begin{abstract}
Spectroscopic ellipsometry (SE) was shown to be an accurate in situ method for determining the composition and thickness of III-V semiconductor layers during growth. In order to fully exploit the potential of SE for real-time in situ control, one needs to acquire a database of optical constants. In this article, we present the acquisition and parameterization (both composition and temperature) of a fully dynamic database and its use in the real-time composition control of InAlAs grown on InP. This is accomplished by acquiring SE data from growing films of different compositions, while the temperature is controlled using feedback from band-gap thermometry. The layer compositions are assessed by fitting high-resolution x-ray diffraction patterns with a simulation based on dynamical diffraction theory. In order to improve the stability during real-time control, the database was parameterized using a transfer function model. The parameterized database was then used, in real time, during growth to control the $\operatorname{In}_{x} \mathrm{Al}_{1-x} \mathrm{As}$ film composition $(x)$ to within \pm 0.003 . (C) 2000 American Vacuum Society. [S0734-211X(00)05803-0]
\end{abstract}

\section{INTRODUCTION}

Molecular-beam epitaxy (MBE) has been widely used to grow commercial wafers for electronic devices such as high electron mobility transistors (HEMT). It has also been used to grow much more complicated optoelectronic devices such as vertical cavity surface emitting lasers (VCSEL) $)^{1-3}$ and other microcavity devices. ${ }^{4-6}$ In traditional MBE, growths of such complicated structures with high reproducibility on a day to day basis is very difficult. In order to improve the growth reproducibility, substantial research effort has been devoted to the development of in situ sensors capable of providing real-time information about the growing film: temperature, composition, and layer thickness. ${ }^{6-15}$

Following the work of Aspnes, ${ }^{16,17}$ great effort has been devoted to the use of spectroscopic ellipsometry (SE) for in situ monitoring and real-time control of growth. It has been used with success to control, in a feedback loop, the thickness ${ }^{18}$ and alloy composition ${ }^{19-21}$ of the growing films. Moreover, in conjunction with other sensors, it has been proven to yield improved reproducibility in the growths of devices. ${ }^{6,7}$ SE based feedback control is implemented by comparing the measured SE signal of a growing film to a previously acquired database of optical constants (OC), which yields the composition and, provided that interference fringes are present, the thickness of the film. If it is parametrized, ${ }^{22,23}$ the precision of this database is improved over that obtained by straight interpolation between experimental data points. For the composition control of ternary compounds, such as AlGaAs, InGaAs or InAlAs, one of the group III effusion cells is kept at constant temperature during

\footnotetext{
${ }^{a}$ Current address: Bandwidth9, Inc., 46410 Fremont Blvd., Fremont, CA 94538-6409; electronic mail: mbeaudoin@bw9.com

b) Author to whom correspondence should be addressed; electronic mail: yhzhang@asu.edu
}

the entire growth, while the difference between the target and measured compositions is used to change the temperature of the other cell. ${ }^{7,20}$ Hence, the accuracy of the control depends on the accuracy of the database of optical constants against which the growing film is compared. Since SE is surface sensitive, it has been found that the optical constants of a growing layer, which we call the "dynamic" OC, actually differ from those of an "identical" layer just sitting at the growth temperature (under group- $\mathrm{V}$ overpressure) and which we refer to as "static" OC. ${ }^{21}$ Also, because SE uses polarized light, the signal is sensitive to the crystal orientation of the substrate; this dependence can be averaged out by taking measurements under substrate rotation. In this article, we present the acquisition, parameterization and validation of a dynamic OC database for InAlAs alloys with compositions near the lattice-matched condition to InP.

\section{GROWTH AND ACQUISITION OF OC DATABASE}

The experimental setup has been described previously. ${ }^{18,19}$ We use a DCA ${ }^{\mathrm{TM}}$ MBE chamber equipped with ellipsometer ports. Each port makes an angle of $75^{\circ}$ with the normal to the substrate and is equipped with a $\mathrm{BOMCO}^{\mathrm{TM}}$ low birefringence window. On an MBE manipulator, samples are normally mounted with a surface normal at a small angle with the rotation axis. During substrate rotation, this angle processes and is commonly referred to as substrate wobble in MBE jargon. In the $\mathrm{DCA}^{\mathrm{TM}}$ machine, this wobble is minimized through the use of three piezoelectric crystals in the substrate manipulator. The measured SE signal, $\rho$, is given by

$$
\rho=\frac{R_{P}}{R_{S}}=\frac{\left|R_{P}\right|}{\left|R_{S}\right|} e^{i\left(\delta_{P}-\delta_{S}\right)}=\tan (\Psi) e^{i \Delta},
$$




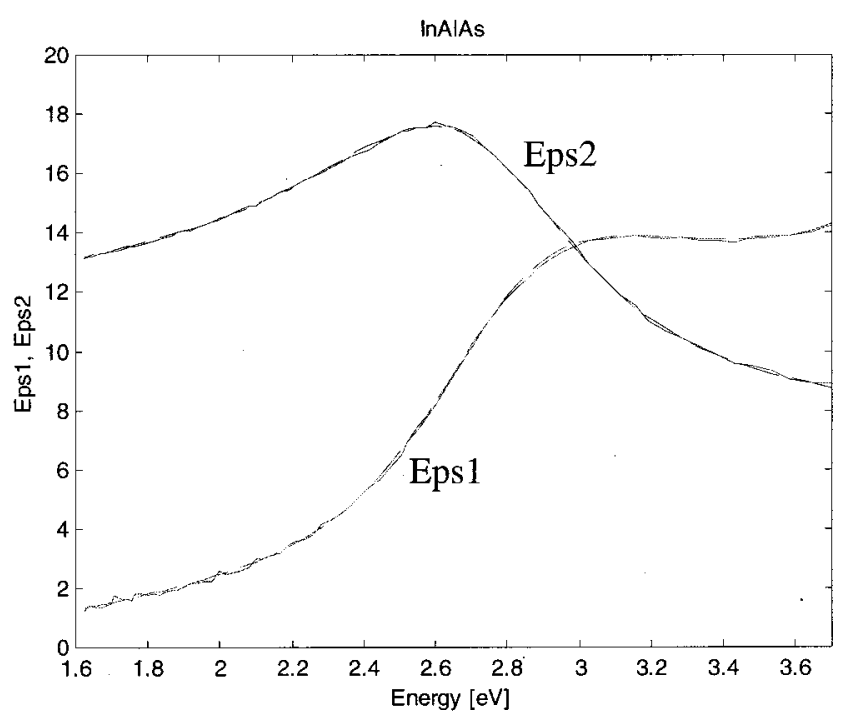

FIG. 1. Typical optical functions of InAlAs showing both the real (Eps1) and imaginary (Eps2) parts. The solid lines are measured by spectroscopic ellipsometry while the dashed lines are from a parameterization using a transfer function model.

where $R_{P}$ and $R_{S}$ are the reflection coefficients for light polarized perpendicular and parallel to the plane of incidence. This is expressed mathematically by the amplitude, $\tan (\Psi)$, and phase, $\Delta$, difference between the $p$ and $s$ components of the electric field. The $\Psi$ and $\Delta$ parameters are directly related to the Fresnel coefficients of the material. A J. A. Woollam M88 ${ }^{\mathrm{TM}}$ ellipsometer and its GrowthManager ${ }^{\mathrm{TM}}$ (GMan) software were used to measured $\Psi$ and $\Delta$ parameters as a function of wavelength ( 88 wavelengths ranging from 277 to 765 $\mathrm{nm})$. The measurement is then analyzed with GMan in order to extract the film's OC. Despite the use of $\mathrm{BOMCO}^{\mathrm{TM}}$ windows, a residual birefringence remains which needs to be corrected for the OC. Fortunately, the latest versions of J. A. Woollam's GMan software is able to accurately measure both the in-plane and out-of-plane window birefringence. ${ }^{24}$ The InAlAs OC database presented here has been corrected for residual window birefringence. The solid lines of Fig. 1 show typical dynamic OC, both real (Eps1) and imaginary (Eps2) parts, for InAlAs along with the parameterization scheme (see below).

Since the SE signal is used to extract the film's OC, it is imperative that the proper angle of incidence be given to GMan; if a wrong angle is used, GMan will return an incorrect value for the OC. Determination of the angle was accomplished by measuring the room temperature SE signal of a bare InP substrate; this SE signal was then compared to an accurate InP OC database acquired ex situ. The substrate temperature was controlled at room temperature using bandgap thermometry. ${ }^{25}$ To improve the reproducibility in angle between runs and to measure the substrate wobble under rotation, a screen was installed in the exit optical path perpendicular to the reflected polarized light. Both the absolute angle and wobble could then easily be measured by looking at the spot mean position and displacement on the screen

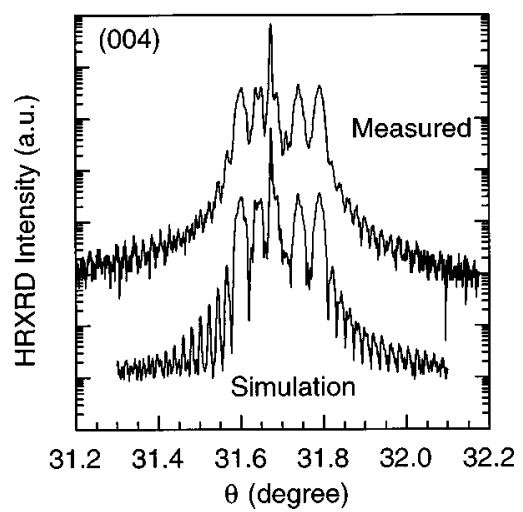

FIG. 2. Symmetric (004) high-resolution x-ray diffraction pattern of a structure consisting of five InAlAs films grown on InP. The bottom pattern is from a simulation using dynamical diffraction theory and is shifted down for clarity. These patterns are used to determine the compositions of the epitaxial layers grown during the acquisition of the optical constant database.

during substrate rotation. Through the use of the three piezoelectric crystals on the substrate manipulator, the sample wobble during the $\mathrm{OC}$ database acquisition was kept below $\pm 0.019^{\circ}$.

The process of acquiring an $\mathrm{OC}$ database consists in growing films of different compositions and temperatures, extracting the $\mathrm{OC}$ and measuring the film composition accurately by an ex situ method. We used optical band edge thermometry ${ }^{25}$ (Thermionics DRS $1000^{\mathrm{TM}}$ system) to control the growth temperature to precision of $\pm 1{ }^{\circ} \mathrm{C}$. The accuracy of this measurement depends on independent calibrations but is typically around $\pm 5^{\circ} \mathrm{C}$ at typical growth temperatures. ${ }^{26}$ The oxide was desorbed from InP wafers under an $\mathrm{As}_{4}$ overpressure up to temperatures of $535^{\circ} \mathrm{C}$. At this point, the reflection high-energy electron diffraction (RHEED) showed a $4 \times 2$ surface reconstruction. The temperature was then lowered to the desired growth temperature and InAlAs was grown directly on InP. Three samples were grown to acquire the dynamic OC database. Each sample consists of five layers with different compositions around the lattice matching condition $(x=0.520)$ ranging from $x=0.490$ to $x=0.536$ (InAs mole fraction). The composition values quoted here assume Vegard's rule and room temperature lattice constants of $0.58684,0.60590$, and $0.56618 \mathrm{~nm}$ for InP, InAs, and AlAs, respectively. Within each sample the growth is carried out at only one temperature, while each sample is grown at a different temperature: 439,475 , and $513{ }^{\circ} \mathrm{C}$. Hence, with three samples, a dynamic OC database consisting of three temperatures and five compositions could be acquired. By stopping the growth between each layer, a complete static OC database consisting of five temperatures and five compositions was also acquired using the first sample.

The film compositions were determined ex situ using high-resolution x-ray diffraction (HRXRD) and reciprocal space mapping on a Philips ${ }^{\mathrm{TM}}$ five-crystal diffractometer. Figure 2 shows a typical (004) HRXRD patterns where the five InAlAs film peaks are observed on either side of the InP Bragg peak. The composition of each of the five layers is 


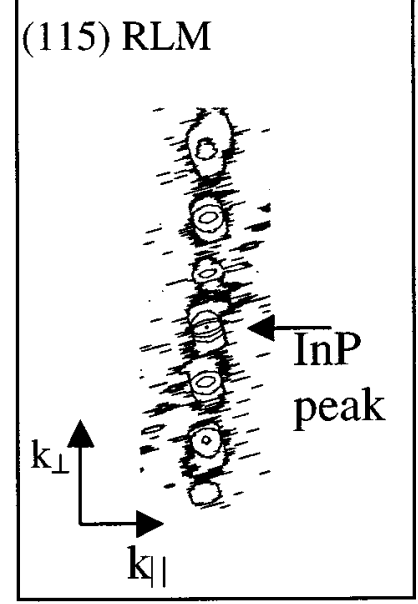

FIG. 3. Asymmetric (115) glancing exit reciprocal lattice map of the sample shown in Fig. 2. The layer peaks are sharp, indicating good crystallinity with low mosaicity, and line up at the same $k_{\|}$value indicating highly coherent unrelaxed InAlAs layers.

extracted by comparing the experimental pattern with a theoretical simulation based on dynamical diffraction theory ${ }^{27}$ and assuming that the film is fully coherently strained (unrelaxed). To ensure this, a (115) reciprocal lattice map (RLM) was also acquired in the glancing exit geometry as shown in Fig. 3 for the sample of Fig. 2. The (115) contours are all perfectly aligned in the $k_{\|}$direction of reciprocal space indicating that the film is coherently strained. The film peaks on the RLM are narrow and sharp; this indicates that the films are of high quality with very little mosaicity.

\section{PARAMETERIZATION AND TESTING OF OC DATABASE}

Several methods for parameterizing the optical functions exist. ${ }^{28-30}$ Some methods are based on physical principles. These attempt to model the optical functions from other known material parameters of which the band gap is the dominant feature in semiconductors. Other methods neglect the underlying physical principles and simply model the optical functions using a purely mathematical approach. Either method has its own advantages and drawbacks. The transfer function (TF) model used here belongs to the second group of models. It emphasizes the robustness and convergence of the fitting algorithm. ${ }^{23,24}$ This TF model is used to determine the temperature $(T)$ and composition $(x)$ from the real-time OC measurements. This is referred to as model inversion. A good fit to the original OC database is essential for accurate $T$ and $x$ prediction since the performance of the inverse model will deteriorate rapidly with the fitting error.

The TF model used here has been presented in detail previously. ${ }^{22,23}$ To summarize, the complex dielectric constant of the material (OC database) is fit using an eight-order transfer function with temperature and composition dependent coefficients. These coefficients are adjusted to minimize the mean square error between the model and the experimental values. The dashed lines in Fig. 1 show typical parameterization fits to both the real and imaginary parts of the

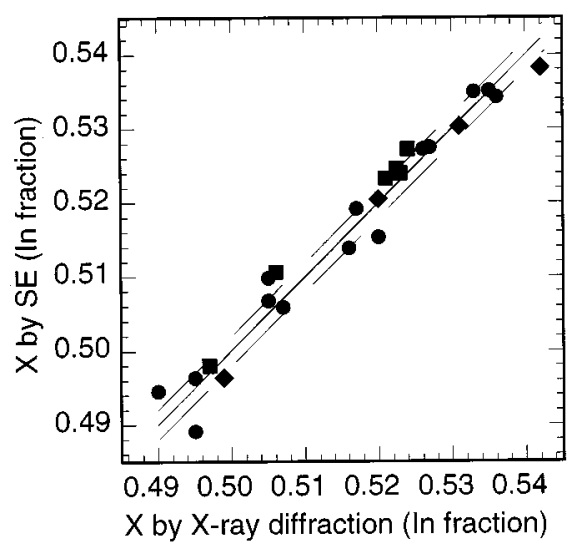

FIG. 4. Comparison of SE and XRD determined In mole fractions $(x)$ in several $\mathrm{In}_{x} \mathrm{Al}_{1-x} \mathrm{As}$ samples. The full squares show the results from the real-time composition control during the growth of InAlAs. The full circles are results from post-growth analyses of the data used to create the OC database while the full diamonds are post-growth analyses on samples not used in the creation of the database. The accuracy of the SE based real-time control is within \pm 0.003 of the In mole fractions using the present parameterized database.

optical functions. The fit is almost indistinguishable from the real data. If the model is used to predict $x$ only while fixing the temperature to the experimentally measured growth temperature an accuracy of \pm 0.0022 (InAs mole fraction) is achieved. When it is used to predict both $T$ and $x$, the accuracy on $x$ improves to \pm 0.00044 with a $T$ accuracy of $\pm 4.9^{\circ} \mathrm{C}$. Presumably, this accuracy would improve if the OC database had been acquired using a finer temperature grid.

In order to verify the accuracy of our OC database and TF model in real time use, the TF model was used to create a parameterized OC database that can be used in the GMan software. GMan was then used to compare the predictions of this database on itself, on previously acquired data and, finally, used in real-time to control the composition of growing InAlAs films. Everytime an experiment is performed, SE data as a function of time are acquired and stored in a file. GMan can be used to analyze the data in real time, as it is acquired, or postgrowth by loading a file of previously stored data. This feature is especially useful to set up the best analysis conditions for real-time feedback control. In all cases, GMan returns composition and thickness values against growth time. Figure 4 shows the average InAlAs composition returned from the SE data analysis of GMan and our parameterized OC database against the composition measured by HRXRD for several samples, film compositions, and growth temperatures. The full circles show the postgrowth analysis of the same data which was used to generate the OC database while the full diamonds show the same type of analysis performed on the SE data of samples acquired prior to the acquisition and parameterization of the database. This exercise is a necessary step if one wishes to use the OC database for real-time feedback control. It allows the operator to set up the fit parameters of the GMan software to obtain rapid convergence and credible results.

The final test of our database consists in using it to control 
the composition of growing InAlAs films. This was accomplished by deliberately choosing an $\mathrm{Al}$ cell temperature which would give an offset from the target composition (every $2{ }^{\circ} \mathrm{C}$ corresponds to $\sim 0.01$ In mole fraction). After approximately $5 \mathrm{~min}$ of growth, at $\sim 1 \mu \mathrm{m} / \mathrm{h}$, the composition values returned by GMan begin to converge to a stable value. We chose to begin feedback control of the Al cell temperature after 8 min of growth using a PID approach. ${ }^{20}$ The full squares of Fig. 4 again show the composition value controlled under feedback control by GMan against the subsequent ex situ composition measured by HRXRD.

In Fig. 4, the full line with a slope of 1 is a best fit to all the data. The dashed lines above and below this line are the \pm 0.002 composition difference with the full line. Of the 24 data points plotted, 16 fall within these lines or $67 \%$. Hence, \pm 0.002 is the standard deviation of the control that can be achieved with our parameterized dynamic OC database on the subset of growth conditions shown here: $0.490 \leqslant x$ $\leqslant 0.540$ (InAs mole fraction) and $439^{\circ} \mathrm{C} \leqslant T$ growth $\leqslant 513{ }^{\circ} \mathrm{C}$. The real-time feedback control experiments were all performed at $475^{\circ} \mathrm{C}$. If we want to include all the data points analyzed or controlled in real time, then the parameterized dynamic OC database allows composition control to within \pm 0.003 of InAs mole fraction in InAlAs closely lattice matched to InP.

\section{CONCLUSION}

A fully dynamic optical constant database has been acquired, parameterized and tested for $\operatorname{In}_{x} \mathrm{Al}_{1-x} \mathrm{As}$ close to lattice-matched composition to InP. The OC database is valid for $0.490 \leqslant x \leqslant 0.540$ and $439^{\circ} \mathrm{C} \leqslant T$ growth $\leqslant 513{ }^{\circ} \mathrm{C}$ and is corrected for window birefringence. The database has been successfully used in a feedback loop to control the composition of InAlAs films to \pm 0.002 in standard deviation or \pm 0.003 maximum deviation from the target composition. This database is publicly available on Arizona State's MBE optoelectronics group website. ${ }^{31}$

\section{ACKNOWLEDGMENT}

This work was supported by DARPA under Contract No. MDA972-95-1-0016 managed by G. S. Pomrenke.

${ }^{1}$ C. Z. Wu, Y. Tsou, and C. M. Tsai, J. Cryst. Growth 201/202, 1005 (1999).

${ }^{2}$ J. Boucart, F. Gaborit, C. Fortin, L. Goldstein, J. Jacquet, and K. Leifer, J. Cryst. Growth 201/202, 1015 (1999).

${ }^{3}$ X. Yang, M. J. Jurkovic, J. B. Heroux, and W. I. Wang, Appl. Phys. Lett. 75, 178 (1999).
${ }^{4}$ J. F. Carlin, P. Royo, M. Ilegems, B. Gerard, X. Marcadet, and J. Nagle, J. Cryst. Growth 201/202, 994 (1999).

${ }^{5}$ S. Orsila, J. Köngäs, M. Toivonen, P. Savolainen, M. Jalonen, and M. Pessa, J. Cryst. Growth 201/202, 985 (1999).

${ }^{6}$ M. Beaudoin, P. Kelkar, M. D. Boonzaayer, W. Braun, P. Dowd, S. R. Johnson, U. Koelle, C.-M. Ryu, and Y.-H. Zhang, J. Cryst. Growth 201/ 202, 990 (1999).

${ }^{7}$ J. Roth, D. H. Chow, G. L. Olson, P. D. Brewer, W. S. Williamson, and B. Johs, J. Cryst. Growth 201/202, 31 (1999).

${ }^{8}$ W. G. Breiland, H. Q. Hou, H. C. Chui, and B. E. Hammons, J. Cryst. Growth 174, 564 (1997).

${ }^{9}$ W. Braun, H. Möller, and Y.-H. Zhang, Appl. Phys. Lett. 74, 138 (1999).

${ }^{10}$ S. A. Chalmers, K. P. Killeen, and E. D. Jones, Appl. Phys. Lett. 65, 4 (1994).

${ }^{11}$ J. Y. Tsao, T. M. Brennan, and B. E. Hammons, J. Cryst. Growth 111, 125 (1991).

${ }^{12}$ K. R. Evans, R. Kaspi, C. R. Jones, R. E. Sherriff, V. Jogat, and D. C. Reynolds, J. Cryst. Growth 127, 523 (1993).

${ }^{13}$ C. H. Kuo, C. Choi, G. N. Maracas, and T. Steimle, J. Vac. Sci. Technol. B 11, 833 (1993).

${ }^{14}$ M. Beaudoin, M. Adamcyk, Z. Gelbart, U. Giesen, I. Kelson, Y. Levy, J. A. MacKenzie, and T. Tiedje, Appl. Phys. Lett. 72, 3288 (1998).

${ }^{15}$ M. Beaudoin, M. Adamcyk, Y. Levy, J. A. MacKenzie, S. Ritchie, T. Tiedje, Z. Gelbart, U. Giesen, and I. Kelson, J. Cryst. Growth 201/202, 28 (1999).

${ }^{16}$ D. E. Aspnes, Thin Solid Films 89, 249 (1982).

${ }^{17}$ D. E. Aspnes, Surf. Sci. 307-309, 1017 (1994).

${ }^{18}$ C.-H. Kuo, M. D. Boonzaayer, M. F. DeHerrera, D. K. Schroder, G. N. Maracas, and B. Johs, J. Cryst. Growth 175/176, 281 (1997).

${ }^{19}$ C.-H. Kuo, M. D. Boonzaayer, M. F. DeHerrera, T. Kyong, Y.-H. Zhang, B. Johs, and J. S. Hale, J. Vac. Sci. Technol. B 16, 1484 (1998).

${ }^{20}$ S. R. Johnson, E. Grassi, M. Beaudoin, M. D. Boonzaayer, K. S. Tsakalis, and Y.-H. Zhang, J. Vac. Sci. Technol. B 17, 1237 (1999).

${ }^{21}$ B. Johs, J. Hale, C. Herzinger, D. Doctor, K. Elliot, G. Olson, D. Chow, J. Roth, I. Ferguson, M. Pelczynski, C. H. Kuo, and S. Johnson, Mater. Res. Soc. Symp. Proc. 502, 3 (1998).

${ }^{22}$ E. Grassi, S. R. Johnson, M. Beaudoin, and K. S. Tsakalis, J. Cryst. Growth 201/202, 1081 (1999).

${ }^{23}$ E. Grassi, S. R. Johnson, M. Beaudoin, and K. S. Tsakalis, J. Vac. Sci. Technol. B 17, 1223 (1999).

${ }^{24}$ Information on the J. A. Woollam ellipsometers can be found at http:// www.jawoollam.com.

${ }^{25}$ S. R. Johnson, C. Lavoie, M. K. Niessen, and T. Tiedje, U.S. Patent No. 5388909 (1995)

${ }^{26}$ M. Beaudoin, A. J. G. DeVries, S. R. Johnson, H. Laman, and T. Tiedje, Appl. Phys. Lett. 70, 3540 (1997).

${ }^{27}$ P. F. Fewster, Philips J. Res. 45, 620 (1984).

${ }^{28}$ C. C. Kim, J. W. Garland, H. Abad, and P. M. Raccah, Phys. Rev. B 45, 11749 (1992), and references therein.

${ }^{29}$ C. C. Kim, J. W. Garland, and P. M. Raccah, Phys. Rev. B 47, 1876 (1993), and references therein.

${ }^{30}$ L. I. Kamlet and F. L. Terry, J. Electron. Mater. 24, 2005 (1995), and references therein.

${ }^{31}$ The ASU OC database can be found on the MBE group's FTP site at http://asumbe.eas.asu.edu. Both the raw and parameterized data for InGaAs and InAlAs (dynamic and static) can be found on this web site in addition to AlAs, GaAs, and AlGaAs OC database. 